\title{
Influence des facteurs agro-écologiques et des herbicides sur le rendement et les caractéristiques technologiques des grains et farines de blés tendres (Triticum aestivum L.) et durs (Triticum durum Desf.)
}

\author{
Ali GARANÉ ${ }^{1}$, Mahamadou SAWADOGO ${ }^{2 *}$, Koussao SOME ${ }^{1}$, Mamadou TRAORÉ ${ }^{3}$, \\ Xésus Petrovich PÉQUÉNIO ${ }^{4}$ \\ ${ }^{I}$ Institut de l'Environnement et de Recherches Agricoles (INERA), CREAFK, \\ Département Production Végétale, 01 BP 470 Ouaga 01, Burkina Faso. \\ ${ }^{2}$ UFR/ Sciences de la Vie et de la Terre. Université de Ouagadougou, \\ Laboratoire de Génétique et de Biotechnologie Végétales BP 7021 Ouaga 03, Burkina Faso. \\ ${ }^{3}$ Institut de l'Environnement et de Recherches Agricoles (INERA), CREAFK, Département \\ Gestion des Ressources Naturelles/Système de Production, 01 BP470 Ouaga 01, Burkina Faso. \\ ${ }^{4}$ Université de L'Amitié des Peuples de la Russie, Département Production Végétale et Phytotechnie Générale, \\ Moscou, 117198, rue Miklouko-Maklaya, 6, Russie. \\ *Auteur correspondant, E-mail: sawadogomahamadou@yahoo.fr
}

\section{RÉSUMÉ}

L'étude met en évidence les effets combinés des conditions écologiques, de la variété et des herbicides 2,4+Lontrel et Quartz super sur les rendements, les caractéristiques technologiques des grains et les propriétés boulangères des farines de blés d'origines écologiques différentes dans la région de Moscou. Pour ce faire, les rendements grains, leurs caractéristiques technologiques et la qualité boulangère des farines mesurés ont montrés que ces blés originaires de la Syrie et du Yémen peuvent avec succès s'adapter à la région de Moscou avec des rendements grains de 3,21-3,43 t/ha (Scham-2), 2,78-3,18 t/ha (Bykhys-1) et 2,64-2,95 t/ha (Homari). L'amélioration de l'état phytosanitaire des semis grâce aux herbicides a permis des gains de production grains en moyenne des blés tendres et durs Lyba, Scham-2, Bykhys-1 et Homari respectivement de 12,2-15,5\%, 5,6$6,8 \%, 9,0-11,1 \%$ et $9,8-11,7 \%$. Indépendamment des herbicides appliqués, les différences observées dans la teneur des blés en protéine, gluten et amidon sont dues à la particularité de chaque variété. Aussi, les blés tendres Lyba et Scham-2 avec respectivement 13,4 et 13,8\% (protéine) et 29,6 et 30,2\% (gluten) sont panifiables tandis que les blés durs Bykhys-1 et Homari avec respectivement 15,7 et 15,5\% (protéines) et $35,4 \%$ (gluten) sont pastifiables.

(C) 2013 International Formulae Group. All rights reserved.

Mots clés: Désherbants, protéine, gluten, qualité boulangère, panifiable.

\section{INTRODUCTION}

Le rendement et la qualité de grain de blé se forment grâce à la réutilisation des substances plastiques accumulées par la plante pendant la phase épiaison-floraison mais surtout aux dépens de la photosynthèse des feuilles de l'étage supérieure de la tige et des autres organes de la plante. En effet, les parties libres de la tige, les gaines, les glumes, les barbes qui conservent plus longtemps la 
chlorophylle sont à mesure de prolonger la photosynthèse après sa destruction dans les feuilles (Triboï et al., 2002; Verma et al., 2004). En d'autre terme, plus les feuilles se conserveront longtemps sur la tige, plus longue sera leur activité pour assurer une productivité élevée de l'épi en grains (Hafsi et al., 2000; Spano et al., 2003). Cependant, à la phase d'épiaison, les feuilles de l'étage supérieur de la tige participent plus au processus de formation des grains que celles de l'étage inférieur qui utiliseront pour la respiration une partie des substances produites (Lawes et al., 1971). Dans tous les cas, pour Souza et al. (2004) et Triboi et al. (2003), la formation de grains dépend des facteurs environnementaux, alors que Ayoub et al. (1994), Slama (2002), Slama et al. (2005), Barbottin et al. (2006) et Bancal et al. (2007) soutiennent que ce sont les facteurs phytosanitaires et génotypiques qui sont déterminants. L'objectif du présent travail est d'évaluer en station et au laboratoire les effets combinés des conditions climatiques, de la variété et des herbicides sur les rendements, les caractéristiques technologiques des grains et les propriétés boulangères des variétés de blés tendres dans la région de Moscou.

\section{MATERIEL ET METHODES \\ Le matériel Végétal}

Le matériel végétal se compose de quatre (4) variétés de blé dont deux (2) de blé tendre (Lyba et Scham-2) et deux (2) de blé dur (Bykhys-1 et Homari). Les caractéristiques essentielles des variétés sont présentées dans le Tableau 1.

\section{Essai en station}

Les expérimentations ont été conduites de 1997-1999 sur des parcelles de l'Institut de Recherches Agronomiques de la région centrale dans la ville d'Odinsk située dans la zone des terres «nichernozèm» de la fédération de Russie. Les précédents culturaux ont été le blé d'hiver triticale + viol (Brassica napis L.) comme engrais vert. Les parcelles ont reçu au labour en automne 45 $\mathrm{kg} / \mathrm{ha}$ de substance active de phosphore $\left(\mathrm{P}_{2} \mathrm{O}_{5}\right)$ sous la forme de super phosphate simple et 70 $\mathrm{kg} / \mathrm{ha}$ de calcium $\left(\mathrm{K}_{2} \mathrm{O}\right)$ sous forme de sel de calcium. Les engrais azotés sont utilisés à des doses de $60 \mathrm{~kg} / \mathrm{ha}$ avant les semis lors du labour au printemps et suivit de $30 \mathrm{~kg} / \mathrm{ha}$ d'azote au tallage sous forme de silicate d'ammonium.

Les normes d'ensemencements pratiquées ont été de 5,0 millions de grains/ha pour chaque variété. La parcelle élémentaire est de $50 \mathrm{~m}^{2}$. Avant les semis, les graines ont été au préalable traitées à l'Ethylmercurchloride (granozan) en raison de $2 \mathrm{~kg}$ de substance par tonne de semences et semées en ligne (16 cm x $4 \mathrm{~cm})$. Le Quartz super (concentré soluble) a été dilué dans l'eau (1,0 1/ha dans 350 1) et appliqué deux jours après semis.

Au tallage des blés, la combinaison 2,4D (dose 1,8 kg/ha m. a.) + Lontrel (dose $0,3 \mathrm{~kg} / \mathrm{ha} \mathrm{m}$. a.) a été employée pour la lutte contre les mauvaises herbes. De même toutes les parcelles de l'essai ont été traitées au Triadimefon (Bajleton) 25\% à la dose de 0,6 $\mathrm{kg} / \mathrm{ha}$ dans $500 \mathrm{l}$ d'eau pour la lutte contre les maladies cryptogamiques (fusarioses, Cercosporioses, septorioses etc.) qui sont responsables d'environ $5-40 \%$ des pertes de récoltes du blé.

Le dispositif expérimental est présenté dans le Tableau 2. Il comprend un Split plot à quatre (4) répétitions avec en parcelles principales les variétés de blé et en parcelles secondaires les quatre traitements appliqués.

\section{Les paramètres mesurés}

Dans cette étude, les paramètres suivants ont été mesurés ou calculés:

Le rendement grains à été calculé et comparé d'une part par traitement et d'autre part par variété et par année de culture afin d'apprécier le potentiel de productivité des arêtes de blé dans les conditions climatiques difficiles de 1998 - 1999;

La qualité des grains des différentes variétés de blés a été appréciée par la teneur en azote, protéine et gluten à partir du matériel de semis du blé Lyba et des grains commercialisables de toutes les variétés de blés étudiés; Kjeldahl (Stewart et al., 1963), consistant à doser l'ammoniaque après 
l'hydrolyse de l'azote organique. Cette valeur est multipliée ensuite par un coefficient de transformation de 5,7 pour le blé compte tenue de sa composition moyenne en acides aminés).

Les analyses de laboratoires ont porté sur les caractéristiques technologiques des grains et les propriétés boulangères des farines des différentes variétés de blé selon la méthode éprouvée du laboratoire National Centrale des analyses des qualités technologiques des céréales et produits céréaliers de l'Université de Moscou.

Des analyses de variance, HCPO (Hierarchical Column Prime Order) ont été effectuées pour apprécier si les différences entre les résultats obtenus sont significatives à la probabilité de $5 \%$.

Tableau 1: Les caractéristiques essentielles des variétés de blé.

\begin{tabular}{|c|c|c|c|c|c|}
\hline Type de blé & Variété & Origine & Rendement & Cycle & Caractéristiques essentielles \\
\hline \multirow[t]{2}{*}{$\begin{array}{l}\text { Blés tendres } \\
(\operatorname{Tr} . \text { aestivum L.) }\end{array}$} & Lyba & Russie & $\begin{array}{c}4,0-5,0 \text { t/ha et } \\
\text { plus }\end{array}$ & $\begin{array}{l}\text { Semi - } \\
\text { tardif }\end{array}$ & $\begin{array}{l}\text { Epi non aristé et glabre; } \\
\text { résistant à la rouille brune } \\
\text { (Puccinia triticina) mais } \\
\text { sensible à la rouille jaune } \\
\text { (Puccinia striiformis } \text { West) }\end{array}$ \\
\hline & Scham-2 & Syrie & $4,0-5,0 \mathrm{t} / \mathrm{ha}$ & $\begin{array}{c}\text { Semi - } \\
\text { tardif }\end{array}$ & $\begin{array}{l}\text { Epi aristé et glabre } \\
\text { résistant à la rouille jaune }\end{array}$ \\
\hline \multirow{2}{*}{$\begin{array}{l}\text { Blés durs } \\
\text { (Tr. durum } \\
\text { Desf.) }\end{array}$} & Bykhys-1 & \multirow{2}{*}{ Yémen } & $3,0-4,0 \mathrm{t} / \mathrm{ha}$ & Tardif & $\begin{array}{l}\text { Epi aristé et pubescent; } \\
\text { résistant à la rouille jaune; } \\
\text { sensible au charançon du } \\
\text { grain (Sitophilus granarius) }\end{array}$ \\
\hline & Homari & & $3,0-4,0$ t/ha & Tardif & $\begin{array}{l}\text { Epi aristé, non pubescent; } \\
\text { résistant à la rouille jaune; } \\
\text { sensible au charançon du } \\
\text { grain }\end{array}$ \\
\hline
\end{tabular}

Tableau 2: Schéma expérimental.

\begin{tabular}{|c|c|c|c|c|}
\hline \multirow[t]{2}{*}{ Traitements } & \multicolumn{4}{|c|}{ Variétés de blés } \\
\hline & Lyba & Scham-2 & Bykhys-1 & Homari \\
\hline Témoin & sans herbicides & sans herbicides & sans herbicides & sans herbicides \\
\hline $\begin{array}{l}\text { technologie de base } \\
\text { 2,4-D+Lontrel }\end{array}$ & $\begin{array}{c}\text { 1,8+0,3 kg/ha m.a. } \\
\text { (tallage complet) }\end{array}$ & $\begin{array}{l}\text { 1,8+0,3 kg/ha m.a } \\
\text { (tallage complet) }\end{array}$ & $\begin{array}{r}1,8+0,3 \mathrm{~kg} / \mathrm{ha} \mathrm{m} . \mathrm{a} \\
\text { (tallage complet) }\end{array}$ & $\begin{array}{c}, 8+0,3 \mathrm{~kg} / \mathrm{ha} \\
\mathrm{m} . \mathrm{a} \\
\text { (tallage } \\
\text { complet) }\end{array}$ \\
\hline $\begin{array}{l}\text { Quartz super: } \\
\text { diflufénicanil-50g/1 } \\
\text { isoproturon-500g/1 }\end{array}$ & $\begin{array}{l}\text { 1,0 1/ha m.a. } \\
\text { (prélevée) }\end{array}$ & $\begin{array}{l}\text { 1,0 1/ha m.a. } \\
\text { (prélevée) }\end{array}$ & $\begin{array}{l}\text { 1,0 1 /ha m.a. } \\
\text { (prélevée) }\end{array}$ & $\begin{array}{l}\text { 1,0 1/ha m.a. } \\
\text { (prélevée) }\end{array}$ \\
\hline $\begin{array}{l}\text { Quartz super: } \\
\text { diflufénicanil-50g/1 } \\
\text { isoproturon- } 500 \mathrm{~g} / 1\end{array}$ & $\begin{array}{c}\text { 1,0 } \mathrm{l} / \mathrm{ha} \text { m.a. } \\
\text { (tallage complet) }\end{array}$ & $\begin{array}{c}\text { 1,0 1/ha m.a. } \\
\text { (tallage complet) }\end{array}$ & $\begin{array}{c}\text { 1,0 1/ha.m.a. } \\
\text { (tallage complet) }\end{array}$ & $\begin{array}{l}\text { 1,0 1/ha.m.a } \\
\text { (tallage } \\
\text { complet) }\end{array}$ \\
\hline
\end{tabular}




\section{RESULTATS}

L'influence de la variété et des herbicides sur les rendements grains des blés tendres (Tr. aestivum L.) et durs (Tr. durum Desf.) en fonction des années

Les rendements grains des blés en fonction des traitements sont présentés dans le Tableau 3. De manière générale, les résultats $\mathrm{du}$ Tableau 3 montrent que tous les traitements améliorent les rendements grains du blé des différentes variétés. En effet on peut constater que les traitements augmentent les rendements de la variété Lyba en moyenne de 12,20\% (2,4-D + Lontrel en post-levée) à $15,50 \%$ (quartz super en prélevée), soit un gain de rendement de 0,34 à 0,43 t/ha. De même, il ressort que les traitements quartz super en prélevée et post levée donnent des rendements grains supérieurs chez toutes les variétés mais les meilleurs rendements sont obtenus avec le quartz super en prélevée.

Les rendements grains moyens des trois années montrent une suprématie des variétés Lyba et Scham-2 qui enregistrent de forts rendements allant de 2,78-3,21 t/ha (Lyba) à 3,21-3,43 t/ha (Scham-2).

Le blé dur Bykhys-1 a un rendement sensiblement égal au blé tendre Lyba mais reste cependant inferieur au blé aristé Scham2, tandis que le blé dur Homari présente les plus faibles rendements au cours de l'étude. Néanmoins, on peut constater que durant les mauvaises années caractérisées par des basses températures et un déficit hydrique (19981999), ce sont les variétés de blés durs Bykhys-1 et Homari qui présentent les meilleurs rendements. Cela peut s'expliquer par une meilleure adaptation de ces variétés qui ont mieux réagi aux mauvaises conditions climatiques en cours. Par ailleurs ces variétés (Bykhys-1 et Homari) sont caractérisées par la présence des arêtes (Tableau 1).

L'Influence des herbicides sur la qualité (la teneur en azote, protéine et gluten) des grains à partir du matériel de semis du blé Lyba et des grains commercialisables des autres variétés étudiés

Le Tableau 4 révèle que les différents traitements en herbicide n'ont pas eu d'impact sur les teneurs en protéine, en gluten et en amidon des différents blés. Par contre des différences sont observées entre les types de blé (tendre ou dur); ceci serait dû à la particularité biologique de chaque blé.

A la lecture du tableau, on note également que les blés tendres Lyba et Scham-2 se sont caractérisés par des taux élevés en protéine ; soit respectivement 13,4\% et $13,8 \%$; pendant que leur teneur en gluten $(29,6$ et $30,2 \%)$ est supérieure au standard $(26,0 \%)$.

Les blés durs (Bykhys-1 et Homari) enregistrent des teneurs de $15,7 \%$ et $15,5 \%$ en protéine, alors que leur pourcentage en gluten $(35,4 \%)$ est identique. Par ces caractéristiques, ces blés se sont montrés supérieurs aux blés tendres (Lyba, scham-2) avec respectivement 2,6 -2,7\% pour la protéine et 5,6-5,8\% pour le gluten. Par ailleurs aucune différence significative pour la teneur en amidon n'a été observée entre les blés tendres et durs.

Analyses chimiques et tests technologiques des grains et propriétés boulangères des différentes variétés de blés tendres (Tr. aestivum L.) et durs (Tr. durum Desf.)

Afin de comprendre l'impact de la taille des grains sur certaines caractéristiques essentielles du blé, les grains au centre des épis obtenus manuellement avant la récolte de la variété Lyba sont passés à travers des grilles (trémie) de diamètre 2,5-3,0 $\mathrm{mm}$ afin d'obtenir un échantillon de matériel de semis composé de gros grains (diamètre 2,5-3,0 $\mathrm{mm}$ ) pour l'analyse tandis que les grains de calibre inferieur à $2,5 \mathrm{~mm}$ sont classés comme grains commercialisables. Pour les autres variétés, toutes les graines de taille différentes sont mises ensemble pour constituer les grains commercialisables. Après la récolte, les petits grains et légers de diamètre inférieur à $2,5 \mathrm{~mm}$ sont exclus de l'échantillon pour être utilisés pour la cuisson du pain.

Nous nous sommes alors donnés pour objectif de vérifier l'effet du "Dicotomia matricalis" sur les caractéristiques technologiques du grain et la qualité boulangère des farines. Ce processus a été évoqué par Lamarque J.B. en 1778 dans son 
œuvre "la flore française," comme un processus biologique, physiologique et héréditaire qui caractérise la propriété de certains organes individuels de la plante à se développer aux dépens des autres. Cet auteur estime que dans le cas de l'épi, cela aboutit à la formation des grains de différents calibres (petits, moyens et gros) selon l'emplacement sur le rachis (partie inférieure, centrale et supérieure).

Le Tableau 5 présente l'analyse du matériel de semis de la variété de blé Lyba mais aussi des grains commercialisables de l'ensemble des variétés des blés de l'essai.

Les données du Tableau 5 montrent que la variété lyba est caractérisée par des semences de diamètre 2,5-3,0 $\mathrm{mm}$, un poids de 1000 grains très élevé $(41,1 \mathrm{~g})$ et un poids spécifique de $800 \mathrm{~g} / \mathrm{l}$. Ce qui le place dans la catégorie de blé de classe I. Cependant, il est rétrogradé en sous-classe III de par la vitrosité de ses grains (54\%). Par contre, si l'on se base sur certaines de ses caractéristiques technologiques comme la qualité du gluten (PS-75), la force boulangère (W-284), la viscosité de la pâte (80), la capacité de mélange de la farine (52\%), le volume de pain $\left(1060 \mathrm{~cm}^{3}\right)$, le temps de chute Hagberg $(\mathrm{Hg}$ $265 \mathrm{~s})$ et la note boulangère générale $(4,3$ bals), ce blé peut être classé comme un blé fort ; c'est-à-dire un blé de meilleure qualité.

Dans cette étude, notre intérêt s'est beaucoup porté surtout sur les propriétés technologiques des grains et sur la qualité boulangère des farines des grains commercialisables de variétés de blés tendres et durs.

Ainsi, les données du Tableau 5 révèlent que le poids de 1000 graines de la variété Lyba est supérieur de $10.5 \%$ à celui de la variété Sham-2 pendant qu'elle a enregistré un poids spécifique de $20 \mathrm{~g} / \mathrm{l}$, soit $2,6 \%$ inférieur à celui du blé Scham-2. Aussi grâce à la grande homogénéité de ses grains (790 g/l) la variété Scham-2 est en mesure de fournir la même extraction de farine que le blé Lyba. Ces deux blés tendres se placent dans la classification russe en type I. Cependant selon la vitrosité générale de leurs grains $(59 \%$ et $63 \%$ ), la variété Lyba est rétrogradée en sous- type III et Scham-2 en sous-type II. Par ailleurs, une comparaison de ces deux blés pour la qualité du gluten (85), la capacité de mélange de la farine (57\%) et le temps de chute de Hagberg (228 s), la variété Sham-2 s'est montrée plus performante que la variété témoin Lyba.

Selon leurs qualités boulangères (pouvoirs d'hydratation et volumes des pains), les deux variétés ont les caractéristiques de bons blés (hydratation $56,7 \%$ et $62,7 \%$; volumes des pains $970 \mathrm{~cm}^{3}$ et $990 \mathrm{~cm}^{3}$ ).

Concernant les blés durs, il ressort que de par leurs caractéristiques technologiques les variétés Bykhys-1 et Homari sont de type II et du sous-type I par la vitrosité générale des grains ( $89 \%$ et $86 \%$ ).

Les poids spécifiques des deux blés $(758 \mathrm{~g} / \mathrm{l}$ et $751 \mathrm{~g} / \mathrm{l})$ indiquent une bonne maturation des grains, ce qui présage des taux d'extraction de farine élevés. Cependant le poids de 1000 grains légèrement élevé chez la variété Bykhys-1(41,1 g) par rapport au blé Homari (37,8 g) pourrait lui assurer de meilleures propriétés meunières.

Les deux blés sont sensiblement égaux en protéines $(15,7 \%$ et $15,5 \%)$ et identiques par la valeur du gluten $(35,4 \%)$ dont la qualité est du premier groupe chez les deux blés (70 et 75), déterminant ainsi des propriétés physiques suffisamment élevées pour cette variété. Ces bonnes caractéristiques sont confirmées à l'alvéographe de Chopin par les valeurs du $\mathrm{P} / \mathrm{L}$ (1,8 $\mathrm{mm}$ et 1,6 mm) et du W (299 j).

Les semoules de ces blés durs (Bykhys1 et Homari) possèdent des taux d'hydratation élevés $(71,2 \%$ et $71,6 \%)$ et des volumes de pain $\left(970 \mathrm{~cm}^{3}\right.$ et $\left.940 \mathrm{~cm}^{3}\right)$ importants. Cependant, les valeurs élevées des indices de viscosité (105 U.F. et 115 U.F.) associés à l'insuffisance de la capacité de mélange des farines $(54 \%)$ ont rabaissés leurs qualités boulangères qui caractérisent cette espèce de blé. Dans l'ensemble, ces deux variétés de blés durs peuvent avec succès servir de blés pastifiables. 
A. GARANÉ et al. / Int. J. Biol. Chem. Sci. 7(3): 1210-1220, 2013

Tableau 3 : L'influence des herbicides et des particularités biologiques des variétés sur les rendements grains des blés tendres (Tr. aestivum L.) et durs (Tr. durum Desf.).

\begin{tabular}{|c|c|c|c|c|c|c|}
\hline \multirow[t]{2}{*}{ Traitements } & \multicolumn{3}{|c|}{ Rendements; (t/ha) } & \multicolumn{3}{|c|}{ Gain rendement/témoin } \\
\hline & 1997 & 1998 & 1999 & moyenne & t/ha & $\%$ \\
\hline \multicolumn{7}{|c|}{ Variété de blé tendre (Tr. aestivum L.): Lyba } \\
\hline Témoin: sans herbicides & 4,04 & 2.97 & 1,32 & 2,78 & - & - \\
\hline 2,4-D + Lontrel en post-levée & 4,35 & 3,25 & 1,75 & 3,12 & $+0,34$ & 12,20 \\
\hline Quartz super en prélevée & 4,37 & 3,27 & 1,99 & 3,21 & $+0,43$ & 15,50 \\
\hline Quartz super en post-levée & 4,41 & 3,31 & 1,89 & 3,20 & $+0,42$ & 15,20 \\
\hline $\mathrm{HCP}_{05}$ & 0,16 & 0,13 & 0,19 & 0,12 & - & - \\
\hline \multicolumn{7}{|c|}{ Variété de blé tendre (Tr. aestivum L.): Scham-2 } \\
\hline Témoin: sans herbicides & 4,15 & 3,41 & 2,07 & 3,21 & - & - \\
\hline 2,4-D+Lontrel en post-levée & 4,27 & 3,57 & 2,33 & 3,39 & $+0,18$ & 05,60 \\
\hline Quartz super en prélevée & 4,33 & 3,59 & 2,37 & 3,43 & $+0,22$ & 06,80 \\
\hline Quartz super en post-levée & 4,35 & 3,57 & 2,34 & 3,42 & $+0,21$ & 06,50 \\
\hline $\mathrm{HCP}_{05}$ & 0,14 & 0,11 & 0,17 & 0,11 & - & - \\
\hline \multicolumn{7}{|c|}{ Variété de blé dur (Tr. durum Desf.): Bykhys-1 } \\
\hline Témoin: sans herbicides & 3,37 & 2,59 & 2,67 & 2,87 & - & - \\
\hline 2,4-D +Lontrel en post-levée & 3,55 & 2,88 & 2,98 & 3,13 & $+0,26$ & 09,00 \\
\hline Quartz super en prélevée & 3,59 & 2,89 & 3,09 & 3,19 & $+0,32$ & 11,10 \\
\hline Quartz super en post-levée & 3,56 & 2,90 & 3,04 & 3,16 & $+0,29$ & 10,10 \\
\hline $\mathrm{HCP}_{05}$ & 0,11 & 0,17 & 0,13 & 0,13 & - & - \\
\hline \multicolumn{7}{|c|}{ Variété de blé dur (Tr. durum Desf.): Homari } \\
\hline Témoin: sans herbicides & 3,39 & 2,27 & 2,46 & 2,64 & - & - \\
\hline 2,4-D + Lontrel en post-levée & 3,31 & 2,62 & 2,78 & 2,90 & $+0,26$ & 09,84 \\
\hline Quartz super en prélevée & 3,34 & 2,65 & 2,81 & 2,93 & $+0,29$ & 10,90 \\
\hline Quartz super en post-levée & 3,33 & 2,73 & 2,79 & 2,95 & $+0,31$ & 11,70 \\
\hline $\mathrm{HCP}_{05}$ & 0,12 & 0,18 & 0,14 & 0,12 & - & - \\
\hline
\end{tabular}


Tableau 4: L'influence des herbicides et des particularités biologiques des blés tendres (Tr. aestivum L.) et durs (Tr. durum Desf.) sur la qualité des grains.

\begin{tabular}{|c|c|c|c|c|c|}
\hline \multirow[b]{2}{*}{ Traitements } & \multicolumn{4}{|c|}{ Teneur; (\%) } & \multirow{2}{*}{$\begin{array}{l}\text { Poids } \\
\text { spécifique } \\
\text { (g/l) }\end{array}$} \\
\hline & azote & Gluten & Protéine & Amidon & \\
\hline \multicolumn{6}{|c|}{ Variété de blé tendre (Tr. aestivum L.): Lyba } \\
\hline \multirow{3}{*}{$\begin{array}{l}\text { Témoin sans herbicide } \\
\text { 2,4-D +Lontrel en post-levée } \\
\text { Quartz super en prélevée }\end{array}$} & 2,37 & 29,60 & 13,50 & 64,4 & 772 \\
\hline & 2,35 & 29,60 & 13,40 & 64,3 & 772 \\
\hline & 2,37 & 30,00 & 13,50 & 64,5 & 772 \\
\hline Quartz super en post-levée & 2,37 & 29,90 & 13,50 & 64,4 & 772 \\
\hline \multicolumn{6}{|c|}{ Variété de blé tendre (Tr. aestivum L.): Scham-2 } \\
\hline \multirow{3}{*}{$\begin{array}{l}\text { Témoin: sans herbicides } \\
\text { 2,4-D + Lontrel en post-levée } \\
\text { Quartz super en prélevée }\end{array}$} & 2,40 & 29,80 & 13,70 & 64.7 & 792 \\
\hline & 2,42 & 30,20 & 13,80 & 64.6 & 792 \\
\hline & 2,39 & 29,90 & 13,60 & 64.8 & 792 \\
\hline Quartz super en post-levée & 2,39 & 30,10 & 13,60 & 64.6 & 792 \\
\hline \multicolumn{6}{|c|}{ Variété de blé dur (Tr. durum Desf.): Bykhys-1 } \\
\hline \multirow{4}{*}{$\begin{array}{l}\text { Témoin: sans herbicides } \\
\text { 2,4-D +Lontrel en post-levée } \\
\text { Quartz super en prélevée } \\
\text { Quartz super en post-levée }\end{array}$} & 2,77 & 35,40 & 15,80 & 62.7 & 758 \\
\hline & 2,75 & 35,20 & 15,70 & 62.5 & 758 \\
\hline & 2,77 & 35,30 & 15,80 & 62.6 & 758 \\
\hline & 2,77 & 35,40 & 15,80 & 62,7 & 758 \\
\hline \multicolumn{6}{|c|}{ Variété de blé dur (Tr. durum Desf.): Homari } \\
\hline \multirow{4}{*}{$\begin{array}{l}\text { Témoin: sans herbicides } \\
\text { 2,4-D + Lontrel en post-levée } \\
\text { Quartz super en prélevée } \\
\text { Quartz super en post-levée }\end{array}$} & 2,79 & 35,60 & 15,90 & 62,5 & \multirow{4}{*}{$\begin{array}{l}752 \\
752 \\
752 \\
752\end{array}$} \\
\hline & 2,77 & 35,40 & 15,80 & 62,7 & \\
\hline & 2,75 & 35,30 & 15,70 & 62,6 & \\
\hline & 2,77 & 35,40 & 15,80 & 62,7 & \\
\hline
\end{tabular}

Tableau 5: Résultats des analyses chimiques et tests technologiques sur les variétés de blés tendre (Tr. aestivum L.) et durs (Tr. durum Desf.).

\begin{tabular}{|c|c|c|c|c|c|}
\hline \multirow{2}{*}{ Caractéristiques technologiques } & \multirow{2}{*}{$\begin{array}{c}\text { Lyba (semence) } \\
2,5-3 \mathrm{~mm}\end{array}$} & \multicolumn{4}{|c|}{ grains commercialisables } \\
\hline & & Lyba & Scham-2 & Bykhys-1 & Homari \\
\hline \multicolumn{6}{|l|}{ Grains } \\
\hline Poids 1000 grains (PMG); (g) & 41,1 & 37,6 & 34,0 & 41,1 & 37,8 \\
\hline Poids spécifique; (g/l) & $\begin{array}{l}41,1 \\
800\end{array}$ & 772 & 790 & 758 & 752 \\
\hline Vitrosité générale; (\%) & 54 & 52 & 63 & 89 & 86 \\
\hline Protéine; (\%) & $\begin{array}{c}54 \\
13,2\end{array}$ & 14,4 & $\begin{array}{c}03 \\
13,6\end{array}$ & $\begin{array}{c}89 \\
15,7\end{array}$ & $\begin{array}{c}80 \\
15,5\end{array}$ \\
\hline \multicolumn{6}{|l|}{ Farines } \\
\hline Gluten humide; $(\%)$ & 29,6 & 30,5 & 29,8 & 35,4 & 35,4 \\
\hline PS (Qualité de gluten) & 75 & 80 & 85 & 70 & 75 \\
\hline Elasticité du test; (mm) & 71 & 52 & 78 & 121 & 122 \\
\hline P/L (élasticité/extensibilité), mm & 0,5 & 0,4 & 0,7 & 1,8 & 1,6 \\
\hline W (Force boulangère); Joul & 284 & 243 & 221 & 299 & 299 \\
\hline Taux d'hydratation; (\%) & 57,5 & 56,7 & 62,7 & 71,2 & 71,6 \\
\hline \multicolumn{6}{|l|}{ Temps de formation d'une } \\
\hline pâte consistante; (mn) & 2,0 & 2,0 & 3,0 & 2,5 & 2,5 \\
\hline Test de viscosité; U.F. & 80 & 110 & 100 & 105 & 115 \\
\hline Capacité de mélange des farines $(\%)$ & 52 & 49 & 57 & 54 & 54 \\
\hline \multicolumn{6}{|l|}{ Pains } \\
\hline Amylographe Brabender $\left(\mathrm{cm}^{3}\right)$ & 1060 & 970 & 990 & 970 & 940 \\
\hline Temps chute Hagberg (s) & 265 & 80 & 228 & 261 & 251 \\
\hline Valeur boulangère (bals) & 4,3 & 3,7 & 3,8 & 4,0 & 4,0 \\
\hline
\end{tabular}




\section{DISCUSSION}

En dehors des herbicides appliqués et de la plasticité des blés étudiés (l'adaptation aux conditions édaphiques), les arêtes chez les blés ont jouées un rôle prépondérant dans le rendement des grains pendant les années défavorables. Nos résultats confirment ceux de Leden (1974), selon lesquels l'activité photosynthétique des arêtes augmenterait la masse du grain en moyenne de $12,9 \%$ et que les arêtes contribuent a $41 \%$ a l'accumulation de la matière sèche dans les épis tandis que les autres organes végétatifs de la plante (feuilles, tiges, gaines, ligules...) contribuent a $12 \%$ a l'accumulation de la matière sèche.

L'état des organes photosynthétiques de l'épi (rachi, épillets, glumes et arêtes) qui conservent plus longtemps la chlorophylle que les feuilles est essentiel dans la dynamique d'accumulation et le temps réparti à la croissance définitive de la biomasse sèche du grain de blé. Ainsi, des chercheurs (Triboï and al., 2002; Verma et al., 2004) ont fait observer le rôle joué par les écailles des épillets et les barbes dans la formation du grain par la présence en elles de tissus assimilant bien développés. Aussi, selon Fokar et al. (1998), la photosynthèse des écailles assurerait à elle seule un certain pourcentage de la masse de matière sèche du grain au cours des trois premières semaines qui suivent la floraison du blé d'été. Pour Nemmar (1980), Slama (2002) et Slama et al. (2005), le potentiel des barbes dans le rendement par augmentation de la surface photosynthétique de l'épi serait accru dans les conditions chaudes et sèches lorsque la surface foliaire réduite possède une activité moindre et l'intervalle de temps entre l'épiaison et la maturité du blé raccourci.

La qualité (la teneur en azote, protéine et gluten) des graines obtenue indique que les différents traitements en herbicide n'ont pas eu d'impact sur les teneurs en protéine, en gluten et en amidon des différents blés. Par contre, des différences sont observées entre les types de blé (tendre ou dur); ceci serait dû à la particularité biologique de chaque blé. Nos résultats sont en inadéquation avec ceux obtenus par Martin et al. (1990), qui dans leurs travaux ont prouvé que les herbicides Dicamba $+2,4-D$ et Dicamba + MCPA améliorent la teneur des grains en protéine respectivement de 8,5 et $13 \%$. De même, certains herbicides à base d'auxine ont eu un impact positif sur la teneur en protéine des grains de blé sans influencer le taux des acides aminés (lysine et proline). Cependant, dans la littérature, il est reconnu que la teneur en protéines du grain de blé est davantage influencée par le génotype (Otteson et al., 2007; Casagrande et al., 2009) et la fertilisation (Tran et al., 2000; Blackshaw et al., 2005; Manna et al., 2007) que par les pratiques culturales. En pratique selon Bertholdsson et al. (1995), elle est généralement négativement corrélée au rendement.

La taille des graines très importante exprimée par le poids de 1000 graines a eu un impact sur la teneur en protéine des blés de l'étude. A ce propos, des études au champ sur le blé au Montana, ont montré que l'utilisation de grosses semences à forte teneur en protéines par rapport aux petites semences a majoré de $18 \%$ le rendement du blé (Stougaard et Xue, 2004) et a réduit de $25 \%$ la biomasse et la production de graines de folle avoine (Xue et Stougaard, 2002). Les effets combinés de cette pratique avec l'augmentation de la densité de semis selon Stougaard et Xue (2005), ont permis une hausse de $30 \%$ du rendement global du blé. Cependant, en absence de mauvaises herbes, la valeur des plus grosses semences peut ne pas revêtir autant d'importance.

La qualité boulangère des farines des variétés étudiées exprimée à travers les analyses chimiques, les tests technologiques des grains et les propriétés boulangères montre que les grains de nos variétés sont riches en protéines. En effet, il est reconnu que plus un grain de blé est riche en protéines, plus sa farine produira un pain volumineux (Cauvain, 2003). Cependant, certains chercheurs ont démontré que des grains de blé ayant une plus faible teneur en protéines procuraient un pain aussi volumineux que des 
grains à plus haute teneur en protéines (Kihlberg et al., 2004; Thomsen et al., 2008). Pour Fowler et al. (2004), en deçà de 12,5\% de protéines, le potentiel panifiable devient difficilement prévisible. Ces discordances illustrent l'importance de combiner selon les auteurs (Gupta et al., 1992; Khatkar et al., 2002; Al-Eid, 2006), les analyses physicochimiques des grains (teneurs en protéines et en gluten...) avec les tests de qualité boulangère de la farine (capacité d'absorption d'eau de la farine, volume du pain) lorsque des investigations approfondies sur le contrôle de la qualité du blé sont conduites. D'autre part, pour les auteurs Ayoub et al. (1994) l'indice de chute de Hagberg est un autre critère important de qualité panifiable établi dans de nombreux pays. Davantage influencé par le génotype que par les pratiques culturales, son seuil bas, selon le CCG. (2009) indique que l'amidon a déjà été dégradé dans le grain en sucre qui peut être le signal de la germination des grains sur l'épi avant la récolte. Une telle farine retient moins d'eau, donne une pâte collante (forte viscosité) difficile à manier et des pains moins volumineux et déformés.

\section{Conclusion}

L'étude a pour but de mettre en évidence les effets combinés des conditions écologiques, de la variété, mais aussi des herbicides 2,4-D + Lontrel et quartz super sur les rendements des blés, les caractéristiques technologiques des grains et les propriétés boulangères des blés d'origines écologiques différentes dans la région de Moscou.

Pour cela, le rendement grains calculé et comparé d'une part par traitement et d'autre part par variété et par année de culture a permis d'apprécier le potentiel de productivité des arêtes des blés durs aristés (Bykhys-1 et Homari) dans les conditions climatiques difficiles (1998 - 1999) qui ont formé un rendement plus stable au cours de l'étude indépendamment des traitements. La qualité de grains des différentes variétés de blés appréciée par la teneur en azote, protéine et gluten a révélé les particularités de chaque variété indépendamment des herbicides employés. Les analyses physico-chimiques des grains et les tests de qualité boulangère de la farine des différentes variétés de blés ont permis de recommander les blés durs Bykhys1 et Homari comme des blés pastifiables pour la fabrication des pâtes. Cependant, pour l'obtention de ces résultats, en plus des mesures phytosanitaires appliquées (lutte contre les plantes adventices, le charançon du grain de blé, les maladies fongiques..), un apport d'engrais $\mathrm{N}_{60} \mathrm{P}_{70} \mathrm{~K}_{70}$ est nécessaire pour obtenir des rendements élevés de grains dans la zone de notre étude.

\section{RÉFÉRENCES}

Al-Eid SM. 2006. Effect of nitrogen and manure fertilizer on grain quality, baking and rheological properties of wheat grown in sandy soil. Journal of the Science of Food and Agriculture, 86: 205-211.

Ayoub M, Guertin S, Frégeau-Reid J, Smith DL. 1994. Nitrogen fertilizer effect on bread making quality of hard red spring wheat in eastern Canada. Crop Science, 34: 1346-1352.

Bancal MO, Robert C, Ney B. 2007. Modeling wheat growth and yield losses from late epidemics of foliar diseases using loss of green leaf area per layer and pre-anthesis reserves. Annals of Botany, 100: 777-789.

Barbottin A, Le Bail M, Jeuffroy MH. 2006. The Azodyn crop model as a decision support tool for choosing cultivars. Agronomy for Sustainable Development, 26: 107-115.

Bertholdsson NO, Stoy V. 1995. Yields of dry matter and nitrogen in highly diverging genotypes of winter wheat in relation to $\mathrm{N}$-uptake and $\mathrm{N}$-utilization. Journal of Agronomy and Crop Science, 175: 285295.

Blackshaw RE, Molnar LJ, Larney FJ. 2005. Fertilizer, manure and compost effects on weed growth and compétition with winter wheat in western Canada. Crop Protection, 24: 971-980. 
Casagrande M, David C, Valantin Morison M, Makowski D, Jeuffroy MH. 2009. Factors limiting the grain protein content of organic winter wheat in south-eastern France: a mixed-model approach. Agronomy for Sustainable Development, 29: $565-574$.

Cauvain S. 2003. Bread Making: Improving Quality. Woodhead Publishing Limited and CRC Press. LLC: Boca Raton ; 589.

CCG(Commission Canadienne des Grains). 2000. Grains Germés et Grains Fortement Germés. CCG : Winniped.

Fokar M, Nguyen HT, Blum A. 1998. Heat tolerance in spring wheat, Grain filling. Euphytica, 104: 9-15.

Fowler DB, Kovacs MIP. 2004. Influence of protein concentration on farinograph absorption mixing requirements and mixing tolerance. Canadian Journal of Plant Science, 80: 785-772.

Gupta RB, Batey IL, MacRitchie F. 1992. Relationships between protein composition and functional properties of wheat flours. Cereal Chemistry, 69: 125131.

Hafsi M, Mechenneche W, Bouamama L, Djekoune A, Zaharieva M, Monneveux P. 2000. Flag leaf senescence and evaluated by numerical image analysis and its relationship with yield under drought in durum wheat. J. Agron. Crop Sci., 185: 275-280.

Khatkar BS, Fido RJ, Tatham AS, Schofield JD. 2002. Functional properties of wheat gliadins. II. Effects on dynamic rheological properties of wheat gluten. Journal of Cereal Science, 35: 307-313.

Kihlberg I, Johansson L, Kohler A, Risvik E. 2004. Sensory qualities of whole wheat pan bread--influence of farming system, milling and baking technique. Journal of Cereal Science, 39: 67-84.

Lawes DA,Treharne KJ. 1971. Variation in photosynthetic activity in cereal. Euphitica, 20:1.

Ledent JF. 1974. Assimilation et translocation $\mathrm{du} \quad 14 \mathrm{CO}_{2}$ chez le froment (Triticum aestivum L.) en fonction de la densité et de la structure du couvert. Bulletin Sciences Académie Royale Belge, 6: 60.

Manna MC, Swarup A, Wanjari RH, Mishra B, Shahi DK. 2007. Long-term fertilization, manure and liming effects on soil organic matter and crop yields. Soil Tillage Res., 94: 397-409.

Martin DA, Miller SD, Alley HP. 1990. Spring wheat response to herbicides applied at three growth stages. Agron. J., 82(1): 95-97.

Nemmar M. 1980. Contribution à l'étude de la résistance à la sécheresse chez le blé dur (Tr durum Desf.) et chez le blé tendre ( $T r$. aestivum L.): étude l'accumulation de la proline sous l'effet du stress hydrique. Thèse D.A.A. ENSA, Montpellier, p. 63.

Otteson BN, Mergoum M, Ransom JK. 2007. Seeding rate and nitrogen management effects on spring wheat yield and yield components. Agronomy Journal, 99: 615621.

Slama A, Ben Salem M, Ben Naceur M, Zid E. 2005. Les céréales en Tunisie: production, effet de la sécheresse et mécanismes de résistance. Sécheresse, 16(3): 225-229.

Slama A. 2002. Étude comparative de la contribution des différentes parties du plant du blé dur dans la contribution du rendement en grains en irrigué et en conditions de déficit hydrique. Thèse de doctorat en biologie, Faculté des Sciences de Tunis.

Souza EJ, Martin JM, Guttieri MJ, O'Brien KM, Habernicht DK, Lanning SP, McLean R, Carlson GR, Talbert LE. 2004. Influence of genotype, environment, and nitrogen management on spring wheat quality. Crop Science, 44: 425-432.

Spano G, Di Fonzo N, Perrotta C, Platani C, Ronga G, Lawlor DW, Napier JA, Shewry PR. 2003. Physiological characterisation of 'stay green' mutants in durum wheat. J. Exp. Bot., 386: 14151420.

Stougaard RN, Xue Q. 2004. Spring wheat seed size and seeding rate effect on yield 
loss due to wild at (Avena fatua) interference. Weed Science, 52: 133-141.

Stougaard RN, Xue Q. 2005. Quality versus quantity: spring wheat seed size and seeding rate effects on Avena fatua interference, economic returns and economic thresholds. Weed Research, 45: 351-360.

Stuwart BA, Portez LK, Johnson DD. 1963. Immobilization and mineralization of nitrogen in several organic fraction of sol. Soil Sc. Soc. Proc., 27: 302-303.

Thomsen IK, Pedersen L, Jørgensen JR. 2008. Yield and flour quality of spring wheat as affected by soil tillage and animal manure. Journal of the Science of Food and Agriculture, 88: 2117-2124.

Tran TS, Tremblay G. 2000. Recovery of $15 \mathrm{~N}$-labeled fertilizer by spring bread wheat at différent $\mathrm{N}$ rates and application times. Canadian Journal of Soil Science, 74: 279-285.
Triboi E, Martre P, Triboi-Blondel AM. 2003. Environmentally-induced changes of protein composition for developing grains of wheat are related to changes in total protein content. Journal of Experimental Botany, 54: 1731-1742.

Triboï E, Triboï-Blondel AM. 2002. Production and grain or seed composition: a new approach to an old problem-invited paper. European Journal of Agronomy, 16: 163-186.

Verma SP. Sisodiya HS, Awadhesh Kishore, Prakash Chandra. 2004. Effect of oxalates from pearl millet on growth performance in goats' health. Bioved, 15(1-2): 107-110.

Xue Q, Stougaard RN. 2002. Spring wheat seed size and seeding rate affect wild at demographics. Weed Science, 50: 312320. 